\title{
EFEITOS DO PILATES NO SISTEMA CARDIOVASCULAR E METABÓLICO DE MULHERES ATIVAS
}

Karoline Barros Oliveira', Nathalia Tau Maciel Silva', Thaoan Bruno Mariano², Heliard Rodrigues dos Santos Caetano ${ }^{2}$.

Universidade do Oeste Paulista - UNOESTE, Curso de Fisioterapia, Presidente Prudente, São Paulo, Brasil. e-mail: heliard@unoeste.br

\section{RESUMO}

Segundo dados epidemiológicos as taxas de mortalidade vêm progredindo por conta das doenças cardiovasculares (DCV), uma forma de reduzir os riscos é a prática de exercícios físicos, e o Método Pilates vem consolidando-se uma opção. O objetivo deste estudo foi demonstrar os efeitos benéficos proporcionados pela prática do Método Pilates e sua influência sobre parâmetros cardiovasculares e metabólicos em mulheres praticantes. Dentre os resultados a prática do método Pilates observou-se que potencializou o efeito cardioprotetor sobre as alterações cardiovasculares e metabólicas das mulheres, sendo apresentadas alterações significativas $(p<0,005)$ no gasto calórico, frequência cardíaca, frequência respiratória e pressão arterial sistólica do grupo Pilates.

Palavras-chave: Mulheres, Pilates, doença cardiovascular, metabolismo.

\section{PILATES EFFECTS ON CARDIOVASCULAR AND METABOLIC SYSTEM OF ACTIVE WOMEN}

\begin{abstract}
According to epidemiological data, mortality rates have been progressing due to cardiovascular diseases (CVD), one way to reduce risks is the practice of physical exercises, and the Pilates Method has been consolidating one option. The aim of this study was to demonstrate the beneficial effects provided by the practice of the Pilates Method and its influence on cardiovascular and metabolic parameters in women practitioners. Among the results, the practice of the Pilates method potentiated the cardioprotective effect on the cardiovascular and metabolic changes of women, where it showed significant changes $(p<0.005)$ in the energy expenditure, heart rate, respiratory rate and, systolic blood pression of the Pilates group.
\end{abstract}

Keywords: women, Pilates, cardiovascular disease, metabolism.

\section{INTRODUÇÃO}

Uma das principais causas de morte em todo o mundo na população adulta têm sido as doenças cardiovasculares (DCV), dados epidemiológicos expõe que $\mathrm{o}$ índice de mortalidade provindas destas doenças vem progredindo. No Brasil de acordo com dados da OMS esta doença atinge 300 mil pessoas anualmente $^{1}$. Os fatores de riscos podem ser modificáveis como a hipertensão arterial sistêmica (HAS), hiperlipemia, diabetes mellitus (DM), sedentarismo, tabagismo, obesidade, estresse, e os não modificáveis hereditariedade, sexo e idade 2,3 .

A composição corporal dividida em massa magra e massa gorda é um marcador biológico essencial do estado fisiológico e do fornecimento de gorduras no organismo, entretanto a elevação da massa gorda poderá acarretar a evolução das doenças crônicas sendo as mais comuns atualmente a hipertensão arterial sistêmica (HAS), diabetes mellitus (DM), 
obesidade, entre outras 4,5

Fundado por Joseph Pilates, o método Pilates com seu grande destaque sobre o controle do corpo e da mente, vem expandindo o número de adeptos para este método podendo ser realizado em solo ou aparelhos. Sua prática vem minimizando os fatores de risco modificáveis como a hipertensão arterial sistêmica, diabetes, obesidade e sedentarismo, promovendo adaptações neuromusculares, aumentando a massa corpórea magra, força muscular, flexibilidade e equilíbrio que por conta disto vem se consolidando e gerando assim um novo meio de prevenção para DCV e um declínio na taxa de mortalidade ${ }^{6,7}$.

Sabe-se que o exercício físico desempenha um papel positivo sobre a saúde em geral e segundo o American College of Sports Medicine, qualquer individuo deve praticar exercício físico moderado, realizando no mínimo 30 minutos de exercício cinco vezes na semana ${ }^{8,9}$.

Justifica-se a importância da prática de exercícios físicos para melhora da qualidade de vida e a prática do método de Pilates, como uma variável importante capaz de potencializar um efeito cardioprotetor sobre alterações cardiovasculares.

O objetivo é demonstrar os efeitos benéficos diretos e indiretos proporcionados pela prática de exercícios físicos, através do método Pilates e sua influência sob parâmetros cardiovasculares e metabólicos em mulheres adultas praticantes deste método.

\section{METODOLOGIA}

Amostra

Trata-se de um estudo transversal, realizado em um estúdio privado de Pilates na cidade de Presidente Prudente, SP, composto por 40 mulheres, sendo 20 do grupo controle (GC) e 20 do grupo Pilates (GP), com a faixa etária de 18 a 40 anos de idade. O estudo teve como critérios de inclusão mulheres com pelo menos seis meses de prática do método Pilates e os critérios de exclusão mulheres que apresentaram alterações cardiovasculares previas ou a perda de uma das 10 sessões.

Este projeto de pesquisa foi avaliado e aprovado pelo Comitê de Ética em Pesquisa (CEP), vinculado à Comissão Nacional de Ética em Pesquisa (CONEP), segundo a resolução 466/2012 (núm. CAAE. 04525218.4.0000.5515). Os participantes inseridos no estudo foram instruídos em relação a todos os procedimentos executados e assinaram o termo de consentimento livre e esclarecido concordando em participarem do estudo.

Desenho experimental

O estudo foi realizado em um estúdio de Pilates, após o primeiro contato com as participantes foram aplicadas as anamneses e as avaliações físicas, sendo mensurado o peso, estatura e bioimpedância em todas as participantes, já a pressão arterial, frequência cardíaca e frequência respiratória foram realizadas apenas no GP. Após avaliação iniciou-se o treinamento de cinco semanas com o grupo GP, com duas sessões semanais de 60 minutos sendo composta por no máximo quatro voluntárias por vez, totalizando então dez sessões.

\section{Procedimento específico}

A anamnese foi aplicada por meio do questionário SF-36 com as 40 participantes, sendo possível observar os aspectos relacionados à condição da saúde, tais como doenças concomitantes, tabagismo e $\mathrm{o}$ uso de medicamentos diariamente.

$\mathrm{Na}$ avaliação física, o peso corporal foi mensurado por uma balança digital da marca Oxer $^{\circledR}$, onde as participantes vestiam roupas leves, sem adereço e descalças, e a estatura mensurada por uma fita métrica da Vonder $^{\circledR}$ em que as mesmas permaneceram em posição ortostática, descalças com os pés unidos, cabeça ajustada, tendo em seguida como resultado o índice de massa corporal (IMC) que foi calculado com base no peso e estatura das participantes, classificadas segundo a OMS da seguinte forma: baixo peso $\left(<18,5 \mathrm{~kg} / \mathrm{m}^{2}\right)$ peso adequado $(\geq 18,5-25$ $\left.\mathrm{Kg} / \mathrm{m}^{2}\right)$, sobrepeso $\left(\geq 25-30 \mathrm{Kg} / \mathrm{m}^{2}\right)$, obesidade $\left(\geq 30 \mathrm{Kg} / \mathrm{m}^{2}\right)^{10,11}$.

A bioimpedância (BIA) foi avaliada com a marca Biodynamics ${ }^{\circledR}$ modelo 310 e, era realizada no período da manhã, onde as participantes foram orientadas a estarem em jejum, de bexiga vazia e não terem feito atividade física intensa 8 horas antes do exame. Não era realizada em portadores de 
marca-passo e insuficientes cardíacos instáveis, pois alterações no volume poderiam intervir nos resultados ${ }^{12,13}$

A pressão arterial foi aferida com o esfigmomanômetro da marca $\mathrm{Omron}^{\circledR}$, as participantes permaneceram em posição sentada apoiando o braço esquerdo em uma superfície plana à altura do ombro, é importante salientar que antes das sessões todas as mulheres ficaram em um período de 10 min em repouso para normalizar a pressão arterial e ao fim eram verificadas imediatamente após a sessão ${ }^{14}$.

A frequência cárdica foi verificada por um monitor cardíaco da marca Omron $^{\circledR}$, sempre antes e imediatamente após cada sessão. E a Frequência respiratória observavase pelo modo visual, contando durante 60 segundos com o auxílio de um cronometro.

Protocolo de Treinamento

Após as avaliações foram executados 10 exercícios básicos do Método Pilates nas dez sessões (Quadro 1$)^{15}$. Em todas as sessões as voluntárias praticaram exercícios com uma mola de resistência $\mathrm{k}=16,4 \mathrm{kgf} / \mathrm{m}=$ $164 \mathrm{~N}$, esses exercícios eram realizados seguindo os princípios estabelecidos no método Pilates.

Quadro 1. Exercícios básicos do Método Pilates nas dez sessões.

\begin{tabular}{|c|c|}
\hline Respiração Diafragmática & $\begin{array}{l}\text { Em DD, joelhos flexionados e pés apoiados, braços ao longo do } \\
\text { tronco, praticante inspira pelo nariz e expira pela boca. }\end{array}$ \\
\hline $\begin{array}{l}\text { Foot-work Toes (trabalho com a } \\
\text { ponta dos pés) }\end{array}$ & Em DD no Reforme, apoia as pontas dos pés. \\
\hline $\begin{array}{l}\text { Foot-work Hells (trabalho como } \\
\text { calcanhar) }\end{array}$ & $\begin{array}{l}\text { Em DD no Reforme, apoia os calcanhares com os pés em } \\
\text { dorsoflexão. }\end{array}$ \\
\hline $\begin{array}{l}\text { The one leg stretch } \\
\text { (alongamento de uma perna) }\end{array}$ & $\begin{array}{l}\text { Em DD no solo, um joelho flexionado próximo ao tórax, com as } \\
\text { mãos apoiando e o outro estendido. }\end{array}$ \\
\hline $\begin{array}{l}\text { The double leg stretch } \\
\text { (alongamento das duas pernas) }\end{array}$ & $\begin{array}{l}\text { Em DD no solo, joelhos e quadril flexionados em } 90^{\circ} \text {, mãos } \\
\text { apoiadas na lateral das pernas. Inspirar e expirar para estender } \\
\text { MMIl e elevar braços. }\end{array}$ \\
\hline $\begin{array}{l}\text { The leg pull front (extensão } \\
\text { perna para frente) }\end{array}$ & $\begin{array}{l}\text { De quadrúpede com mãos alinhadas com os ombros, estender as } \\
\text { duas pernas e manter o corpo em prancha, expira estendendo uma } \\
\text { das pernas com flexão plantar, e inspirar retornando para o início. }\end{array}$ \\
\hline $\begin{array}{l}\text { Arms pull up and down (levar o } \\
\text { braço para cima e para baixo) }\end{array}$ & $\begin{array}{l}\text { Sentado no Cadilac, MMII cruzados, de costas para a barra irá } \\
\text { segura-lá com as duas mãos supinada, cotovelos e ombros } \\
\text { flexionados a } 90^{\circ} \text {. Inspire para iniciar o movimento e expire } \\
\text { realizando a extensão do ombro flexionando o cotovelo, levando a } \\
\text { barra na direção do peito. }\end{array}$ \\
\hline Variação Arms pull up and down & $\begin{array}{l}\text { Em DD no Cadilac com os joelhos e quadril flexionados. Segure } \\
\text { as alças com as mãos, inspire preparando-se para o movimento, e } \\
\text { na expiração realize a extensão dos ombros. }\end{array}$ \\
\hline Estabilização da escapula & $\begin{array}{l}\text { No Cadilac, na posição de gato na barra torre segure-a com uma das } \\
\text { mãos, mantendo o ombro e cotovelo fletidos. Os joelhos alinhados } \\
\text { com o quadril e a mão de apoio com o ombro. Inspire para iniciar e } \\
\text { expire realizando a extensão do cotovelo. }\end{array}$ \\
\hline Mermaid (sereia) & $\begin{array}{l}\text { Sentado lateralmente no Cadilac, segure a barra torre com ombros } \\
\text { e cotovelos próximos a } 90^{\circ} \text {; as pernas cruzadas movimente a barra } \\
\text { torre para baixo ao mesmo tempo em que flexiona lateralmente o } \\
\text { tronco, levando o MS contralateral acima da cabeça, e retorne a } \\
\text { posição inicial. }\end{array}$ \\
\hline
\end{tabular}

DD - Decúbito Dorsal, MMII - Membros Inferiores, MS - Membro Superior. 
Para mudança de fase foi utilizado o aumento das repetições, as 5 semanas foram divididas em três fases: Fase I: 1a a $2^{a}$ semana com 10 repetições, fase II: 3ạ e 4a semana com 12 repetições e fase III: 5a semana com 15 repetições.

Também foi verificada a percepção do esforço físico das participantes através da escala de OMNI RES ${ }^{16}$ a fim de verificar se a intepretação subjetiva do esforço físico dado por essa escala se correlacionava com o tipo de mola utilizada, pois o exercício de moderado na escala é classificado de 5 a 7, para verificar a carga de externa do treino.

Análise estatística

Os dados foram analisados usando o software estatístico GraphPad Prism. Para análise de normalidade foi aplicado teste de Shapiro Wilk. Para análise pareada foi realizado o teste de ANOVA para medidas repetidas, para comparação entre os grupos o Teste $t$ de Student em caso de distribuição normal ou teste de Wilcoxon para variáveis que não tiveram distribuição Gaussiana. O nível de significância utilizado foi de $5 \%$.

\section{RESULTADOS}

As 40 participantes, foram divididas em dois grupos, sendo 20 do grupo Pilates (GP) e 20 do grupo controle (GC), no qual não houve exclusão de nenhuma delas. Os dados antropométricos foram avaliados nos dois grupos, na primeira e na décima sessão (Tabela 1). Foi possível observar diferença significativa no GP na variável gasto energético $(p<0,0005)$, em razão do aumento no gasto energético nas praticantes do método. Vale ressaltar também que houve diminuição do peso e aumento da massa magra no GP, porém sem alterações significativas, podendo esses resultados estar ligado ao um curto período de coleta.

Tabela 1. Variáveis antropométricas relacionadas aos grupos (GC) e (GP) analisados no estudo na $1^{\circ}$ e $10^{\circ}$ sessão, com resultados em média \pm desvio padrão.

\begin{tabular}{lccc}
\hline Variáveis & GC & GP & p \\
\hline Estatura $(\mathrm{m})$ & $1,65 \pm 0,04$ & $1,64 \pm 0,06$ & 0,570 \\
Peso $(\mathrm{Kg})$ & $65,09 \pm 12,51$ & $63,92 \pm 9,98$ & 0,904 \\
IMC $\left(\mathrm{Kg} / \mathrm{m}^{2}\right)$ & $24,21 \pm 4,18$ & $24,03 \pm 3,70$ & 0,887 \\
Hidratação $(\mathrm{Kg})$ & $47,68 \pm 5,72$ & $50,22 \pm 4,89$ & 0,139 \\
Massa Gorda (Kg) & $34,5 \pm 0,9$ & $34,4 \pm 0,9$ & 0,743 \\
& $(25,3-40)$ & $(23,6-39,8)$ & 0,236 \\
Massa Magra (Kg) & $32,63 \pm 3,844$ & $34,14 \pm 4,06$ & 0,005 \\
Gasto energético (Kcal) & $1776 \pm 170,4$ & $1959 \pm 172,3$ & \\
\hline
\end{tabular}

IMC - índice de Massa Corporal.

Os valores referentes às variáveis cardiovasculares GP (Tabela 2) estão comparando os momentos entre pré e pós de cada sessão nos quais foi encontrada diferença significativa $(p<0,0001)$ entre as medianas da FC, FR, PAS. O aumento das três variáveis durante o exercício físico é justificado, pois as fibras musculares precisam de mais energia, diante disto a FC e FR das praticantes aumentaram para mandar sangue rico em oxigênio $\left(\mathrm{O}_{2}\right)$ para todo o sistema, e consequentemente a PAS aumentou pois o volume de sangue no coração também aumentou. Já a variável PAD como já esperado, não houve alteração pois durante o exercício físico acontece a vasodilatação dos vasos e diminui resistência vascular periférica. 
Tabela 2. Variáveis cardiovasculares relacionadas ao GP, analisadas pré e pós as 10 sessões, com resultados em mediana.

\begin{tabular}{cccc}
\hline Variáveis & GP Pré & GP Pós & $\mathbf{p}$ \\
\hline FC & $67(52-116)$ & $90(59-142)$ & $<0,0001$ \\
FR & $13(11-16)$ & $17(12-20)$ & $<0,0001$ \\
PAS & $120(85-156)$ & $120(95-150)$ & $<0,0001$ \\
PAD & $80(56-100)$ & $80(40-99)$ & 0,3768
\end{tabular}

GP - grupo Pilates, FC - frequência cardíaca, FR - frequência respiratória, PAS - pressão arterial sistólica, PAD pressão arterial diastólica.

\section{DISCUSSÃO}

Os principais resultados deste estudo evidenciaram aumento da frequência respiratória, frequência cardíaca, pressão arterial sistólica, assim como redução do gasto energético, no grupo Pilates após as 10 sessões.

No presente estudo um dos resultados positivos mostrou que o método Pilates tem a respiração como um de seus princípios, que segundo Craig $^{17}$ é um princípio primordial para iniciar a prática deste método. No estudo de Doijad et al. ${ }^{18}$, relataram que as posturas e a respiração empregadas durante o método favorecem o fortalecimento e a resistência dos músculos respiratórios, e assim apresentam melhor performance nas manobras espirométricas de VVM e de CVF, o que também justifica as mudanças apresentadas no presente estudo, além de todos esses benefícios citados em estudos anteriores vale ressaltar, o quanto esse método trabalha o músculo diafragma, tanto por conta da respiração como por contrações dos músculos abdominais, como foi apresentado no estudo de Barbosa et al. ${ }^{19}$, onde diz que durante os exercício do método Pilates toda a dinâmica respiratória é modificada, pois ao realizar a respiração do método, o músculos diafragma encontra resistência pela contração dos músculos abdominais.

Os estudos de Blum ${ }^{20}$, Bertolla et al. $^{21}$, Kolyniak et al. ${ }^{22}$, expressaram o impacto do Pilates para a correção de alterações posturais, aumento da força muscular e da flexibilidade, de modo que tais benefícios podem justificar o ganho de mobilidade, visto que reduzem os possíveis encurtamentos da musculatura respiratória ou melhoram seu alongamento, cooperando para uma eficiente mecânica respiratória. No presente estudo foi possível observar que após as 10 sessões as praticantes saíram mais alongadas e apresentaram melhora da musculatura respiratória que se justifica, pois a base dos exercícios são as respirações corretas, o que auxiliou em mudanças significativas na capacidade respiratória das mulheres do grupo Pilates.

Ao comparar a frequência cardíaca do estudo pré e pós-intervenção tanto em aparelho como em solo apresentou diferenças nos valores, e de acordo com o estudo de Magalhaes et al. ${ }^{23}$, os valores da FC aumentaram após a realização de uma aula de Pilates, porém não chegaram a atingir valores submáximos. Para Leite et al. ${ }^{24}$, ao relacionar os valores da FC do exercício realizado na bola com o executado no aparelho, para o mesmo grupo muscular, observou-se que há diferença significativa, o que mostra que em alguns grupos musculares, exercícios variados podem ter repercussão cardiovascular diversa. No presente estudo foi possível observar que apresentou diferenças significativas na frequência cardíaca, após as dez sessões onde os exercícios eram realizados nos aparelhos e no solo de maneira variada.

Em relação a pressão arterial sistêmica, o presente estudo após avaliação pré e pós-intervenções apresentou diferença significativa na pressão arterial sistólica (PAS) onde observou um aumento, porém não houve mudança significativa na pressão diastólica (PAD) mais a mesma apresentou uma redução, no estudo de Liberalino et al. ${ }^{25}$, que em sua verificação antes e após 20 aulas de Pilates, observou diferença significativa na pressão arterial sistólica (PAS) (antes $118,33 \pm 9,83$ e depois $103,33 \pm 13,66$ 
$\mathrm{mmHg}$ ) e na pressão arterial diastólica (PAD) (início $80 \pm 0,00$ e ao final 78,33 $\pm 11,69$ $\mathrm{mmHg}$. Já Fagard ${ }^{26}$, em 44 estudos randomizados e controlados, analisou a relação exercício e pressão arterial em repouso, encontrando redução da média de pressão sistólica $(3,4 \mathrm{mmHg}$ em geral; 2,6 mmHg em normotensos; 7,4 mmHg em hipertensos) e da pressão diastólica $(2,4 \mathrm{mmHg}$ em geral; 1,8 $\mathrm{mmHg}$ em normotensos; 5,8 $\mathrm{mmHg}$ em hipertensos). E no estudo de Kokkino et al. ${ }^{27}$ relatou reduções de $10 \mathrm{mmHg}$ na pressão sistólica e de 7,5 mmHg na pressão diastólica com treinamento.

Em contrapartida, no estudo de Rocha et al. ${ }^{28}$, não houve modificação estatística em relação às pressões arteriais de mulheres submetidas ao treinamento com o Pilates (antes 102,3 $\pm 10,7$ e depois $101,7 \pm 9,6$ $\mathrm{mmHg}$ ).

A composição corporal tem relação com a porcentagem de gordura nos indivíduos, embora o método Pilates não tenha como objetivo principal o emagrecimento, mulheres vem procurando essa prática com este intuito, no presente estudo pode ser observado a redução do gasto energético após as 10 semanas de prática do método Pilates, segundo o estudo de Caldwell et al. ${ }^{29}$, não foram observadas mudanças significativas na composição corporal de participantes de um programa de exercícios de Pilates. Porém, Jago et al. ${ }^{30}$ constataram melhoras significativas no IMC das mulheres que se submeteram a um programa de exercícios de Pilates. E Arslanoglu et al. ${ }^{31}$ encontraram redução significativa no percentual de gordura corporal de mulheres sedentárias de meia idade que realizaram oito semanas de exercícios regulares de Pilates.

$$
\text { Algumas limitações foram }
$$
encontradas em nosso estudo, como o período limitado para acompanhar as participantes, ou seja, foram realizadas poucas sessões, do qual acredita-se que para modificações cardiovasculares mais significativas, são necessários outros protocolos de exercícios através da utilização do método Pilates, sendo sugerido futuros estudos que contemplem modificações relevantes do sistema cardiovascular.

\section{CONCLUSÃO}

Conclui-se que houve mudanças significativas nos resultados cardiovasculares em mulheres praticantes do método Pilates, assim mostrando um método eficaz e seguro minimizando as chances de desenvolvimento de patologias. Entre os parâmetros antropométricos o gasto energético mostrou-se eficaz associado aos parâmetros cardiovasculares.

\section{AGRADECIMENTOS}

\author{
À Universidade do Oeste Paulista, \\ pelo financiamento dos instrumentos \\ utilizados neste estudo.
}

\section{REFERÊNCIAS}

1. Organização Mundial da Saúde - OMS. Doenças cardiovasculares. 2017.

2. Carvalho CA, Fonseca PCA, Barbosa JB, Machado SP, Santos AM, Silva AAM. The association between cardiovascular risk factors and anthropometric obesity indicators in university students in São Luís in the State of Maranhão, Brazil. Ciênc Saúde Coletiva. 2015;20(2): 479-90. DOI: http://dx.doi.org/10.1590/1413$\underline{81232015202.02342014}$

3. Gus I, Ribeiro RA, Kato S, Bastos J, Medina $C$, Zazlavsky $C$, et al. Variations in the prevalence of risk factors for coronary artery disease in Rio Grande do Sul - Brazil: a comparative analysis between 2002 and 2014. Arq Bras Cardiol. 2015;105(6):573-9. DOI: http://dx.doi.org/10.5935/abc.20150127

4. Ruiz-Montero PJ, Castillo-Rodriguez A, Mikalački M, Nebojsa Č, Korovljev D. 24-weeks Pilates-aerobic and educative training to improve body fat mass in elderly Serbian women. Clin Intervent Aging. 2014;9:243. https://doi.org/10.2147/CIA.S52077

5. Aladro-Gonzalvo AR, Machado-Díaz $M$, Moncada-Jiménez J, Hernández-Elizondo J, Araya-Vargas G. The effect of Pilates exercises on body composition: a systematic review. J Bodyw mov ther. 2012;16(1):109-14. https://doi.org/10.1016/i.jbmt.2011.06.001

6. Marés G, Oliveira KB, Piazza MC, Preis 
Neto C, Bertassoni L. A importância da estabilização central no método Pilates: uma revisão sistemática. Fisioter Mov. 2012;25(2):445-51.

https://doi.org/10.1590/S0103-

\section{2}

7. Jianxiong W. Tan S, Cao L. Exercise training at the maximal fat oxidation intensity improved health-related physical fitness in overweight middle-aged women. J Exerc Sci Fitness. 2015;13(2):111-6. DOI: http://dx.doi.org/10.1016/i.jesf.2015.08.003.

8. Abete I, Goyenechea E, Zulet MA, Martinez JA. Obesity and metabolic syndrome: potential benefitfrom specific nutrition al componentes. Nutr Metab Cardiovasc Dis. 2011;21(2):B1 -15. https://doi.org/10.1016/i.numecd.2011.05.001

9. Malachias MVB et al. Sociedade Brasileira de Cardiologia. 7a Diretriz Brasileira de Hipertensão Arterial. Arq Bras Cardiol. 2016;107(3):1-103.

10. Rodrigues EV et al. Effects of exercise on pain of musculoskeletal disorders: a systematic review. Acta Ortop. Bras. 2014;22(6):334-8. DOI: $\quad$ http://dx.doi.org/10.1590/1413$\underline{78522014220601004}$

11. Ministério da saúde, IMC em adultos, 2017. Disponível em: http://www.saude.gov.br/component/content/a rticle/804-imc/40509-imc-em-adultos

12. Britto EP, Mesquita ET. Bioimpedância elétrica aplicada à insuficiência cardíaca. Rev SOCERJ. 2008;21(3):178-83. Disponível:

http://www.rbconline.org.br/wpcontent/upload s/a2008_v21_n03_a08aatue leonora.pdf 24.

13. Cômodo ARO, Dias ACF, Tomaz BA, Silva-Filho AA, Werustsky CA, Ribas DF, Spolidoro J, Marchini JS. Utilização da bioimpedância para avaliação da massa corpórea. Associação Médica Brasileira e Conselho Federal de Medicina. Projeto Diretrizes, 2009. Disponível em: http://www.abran.org.br/paraprofissionais/diretrizes-abran/

14. Sociedade Brasileira de
Cardiologia. VI Diretrizes Brasileiras de Hipertensão Arterial. 2010. Disponível em: http://publicacoes.cardiol.br/consenso/2010/Dir etriz_hiperten saoassociados.pdf

15. Blog Pilates. 2018. Disponível em: http://www.blogpilates.com.br

16. Robertson RJ, Goss FL, Rutkowisk J, Lenz B, Dixon C, Timmer J. Concurrent validation of the OMNI perceived exertion scale for resistance exercise. Med Sci Sports Exerc. 2003;35(2): 333-41.

17. Craig C. Pilates com a bola. São Paulo: Phorte; 2003.

18. Doijad VP, Surdi AD. Effect of short term yoga practice on pulmonary function tests. Ind J Basic Appl Med Res. 2012;1:226-30.

19. Barbosa AW, Martins FL, Vitorino DF, Barbosa MC. Immediate electromyography changes of the biceps brachii and upper rectus abdominis muscles due to the Pilates centering technique. J Bodyw Mov Ther. 2013;17(3):38590. DOI:

http://dx.doi.org/10.1016/j.jbmt.2013.01.003

20. Blum CL. Chiropractic and Pilates therapy for the treatment of adult scoliosis. J Manipulative Physiol Ther. 2002;25(4):E3. https://doi.org/10.1067/mmt.2002.123336

21. Bertolla $F$, Baroni BM, Junior ECPL, Oltramari JD. Effects of a training program using the Pilates method in flexibility of sub-20 indoor soccer athletes. Rev Bras Med Esporte. 2007;13(4):222-6.

https://doi.org/10.1590/S151786922007000400002

22. Kolyniak IEG, Cavalcanti SMB, Aoki MS. Isokinetic valuation of the musculature involved in trunk flexion and extension: Pilates method effect. Rev Bras Med Esporte. 2004;10(6):491-3.

https://doi.org/10.1590/S1517$\underline{86922004000600005}$

23. Magalhaes $F$, Albuquerque AP, Pyrrho C, Navarro F. Comportamento da pressao arterial e da frequência cardíaca em uma aula utilizando o metodo Pilates. Rev Bras Prescr 
Fisiol Exerc. 2009;3:208-16.

24. Leite TC, Farinatti PTV. Estudo da frequencia cardiaca, pressão arterial e duplo produto em exercicios diversos para grupamentos musculares semelhantes. Rev Bras Prescr Fisiol Exerc. 2003;2:29-49.

25. Liberalino EST, Sousa TCC, Silva VRL. Influência dos exercícios do método Pilates sobre o sistema cardiorrespiratório. REBES. 2013;3(3):59-64.

26. Fagard RH. Exercise characteristics and blood pressure responses to dynamics physical training. Med Sci Sports Exerc. 2001;21(33)484$92 . \quad$ https://doi.org/10.1097/00005768200106001-00018

27. Kokkino PF, Papademetrio V. Exercise and hypertension. Coron Artery Dis. 2000;1(11):99-102.

https://doi.org/10.1097/00019501-200003000$\underline{00002}$

28. Rocha AAO, Costa ACSM, Luduvice FS. Análise da capacidade física e respiratória de alunas praticantes do método pilates no município de Aracaju/SE. Interfaces Científicas - Saúde e Ambiente. 2015;3(2):73-86. https://doi.org/10.17564/2316-

$\underline{3798.2015 v 3 n 2 p 75-86}$

29. Caldwell K, Harrison M, Adams M, Triplett NT. Effect of Pilates and Taiji quan training on self-efficacy, sleep quality, mood, and physical performance of college students. J Bodyw Mov Ther. 2009;13(2):155-63. https://doi.org/10.1016/i.jbmt.2007.12.001

30. Jago $R$, Jonker $M L$, Missaghian $M$, Baranowski T. Effect of 4 weeks of Pilates on the body composition of young girls. Prev Med. 2006;42(3):177-80.

https://doi.org/10.1016/i.ypmed.2005.11.010

31. Arslanoglu E, Cansel A, Behdari R, Ömer S. Effects of eight weeks Pilates exercice on body composition of middle aged sedentary women. Mov Health. 2011;11(1):86-9. 